\title{
Beweglichkeit ist das Gebot der Stunde
}

Agil, also beweglich sollen Mitarbeiter heute arbeiten. Oder „agile“. Ursprünglich kommt der Begriff aus der Softwareentwicklung, wo er dem klassischen Wasserfallmodell entgegengesetzt wurde. Inzwischen wird er aber regelmäßig auch in anderen Zusammenhängen genutzt. Wie so oft bei Modebegriffen wird "agile“ dabei teils zum Allheilmittel gegen alles und zur Wunderwaffe für jeden Zweck erklärt. Und wie immer, ist das in der Realität natürlich nicht der Fall.

Agile Methoden haben praktische Vorteile, sie ermöglichen es, flexibler auf veränderte Rahmenbedingungen zu reagieren oder Erkenntnisse zu berücksichtigen, die sich erst im Verlauf eines Projekts ergeben. Von starren Hierarchien geprägte Organisationen sind meist schlecht darin, solche Bedingungen zu meistern. Agilität macht es möglich, mit dem heutzutage oft dynamischen Marktumfeld adäquat umzugehen. Erst so kann es gelingen, neuen Entwicklungen nicht immer nur hinterherzulaufen. Gleichzeitig gibt es immer noch Fälle, in denen andere Methoden der bessere Ansatz sind. Das gilt zum Beispiel immer dann, wenn es entscheidend wichtig ist, Gesetze und Vorschriften einzuhalten oder wenn eine umfassende Dokumentation nötig ist - Beispiele sind etwa die Pharmaindustrie oder die Medizintechnik.

Problematisch ist dabei, dass die Entscheidung für oder gegen agile Vorgehensweise zu selten auf Grundlage sachlicher Erwägungen getroffen wird. Stattdessen hängt es von den Vorlieben des Managements ab, ob nach Wasserfallmodell oder agil vorgegangen wird. Das führt in der Praxis dann zu Schwierigkeiten. Es ist deshalb von großer Bedeutung, dass

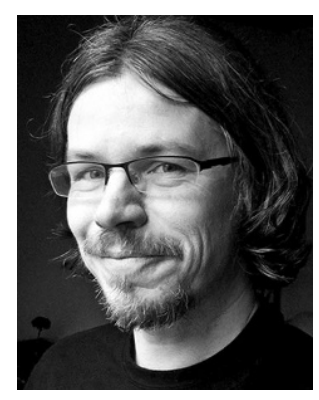

Peter Pagel $(\bowtie)$

peter.pagel@springer.com

Wiesbaden, Deutschland

Manager um die Stärken und Schwächen der verschiedenen Ansätze wissen. Erst dann ist die Grundlage da, um sachbezogen zu entscheiden, wie man an welcher Stelle idealerweise vorgehen sollte.

In diesem Sinne, bleiben Sie beweglich, auch bei der Entscheidung für oder gegen ,agile“.

Herzliche Grüße

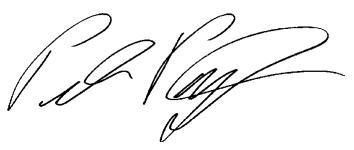

Peter Pagel

Chefredakteur
Wirtschaftsinformatik \& Management 2019 • 11 (4): 181 https://doi.org/10.1365/s35764-019-00200-x

(c) Springer Fachmedien Wiesbaden $\mathrm{GmbH}$, ein Teil von Springer Nature 2019 